\title{
PENECTOMIA E CELIOTOMIA EXPLORATÓRIA EM Chelonoidis carbonarius (SPIX, 1824) - RELATO DE CASO
}

\author{
SILVA, Thassiane Targino ${ }^{1}$; \\ SURITA, Lívia Eichenberg ${ }^{2}$; \\ SANTOS, Eduardo Almeida Ruivo dos ${ }^{3}$; \\ SCHMITT, Bernardo ${ }^{3}$; \\ COSTA, Priscila Medina da ${ }^{2}$; \\ AMORIM, Derek Blaese de ${ }^{4}$; \\ WARTCHOW, Bárbara Schiller ${ }^{3}$; \\ ALIEVI, Marcelo Meller ${ }^{3}$.
}

${ }^{1}$ Médica Veterinária Residente, Medicina de Animais Silvestres, NURFS/CETAS-UFPEL; ${ }^{2}$ Médica Veterinária Residente, Clínica e Cirurgia de Animais Silvestres, UFRGS; ${ }^{3}$ Universidade Federal do Rio Grande do Sul; ${ }^{4}$ Centro de Estudos Costeiros, Limnológicos e Marinhos, Campus Litoral Norte, UFRGS.

\section{RESUMO}

$\mathrm{P}$ ertencentes a Ordem Testudines e Família Testudinidae, os jabutis são répteis de hábitos terrestres, que estão distribuídos por diversos países tropicais, habitando também o Brasil. O jabuti-piranga (Chelonoidis carbonarius) se destaca como espécie frequentemente encontrada em cativeiro, seja por necessidade de manutenção de indivíduos que não podem voltar à vida livre ou por proprietários particulares que os mantém como pet. A manutenção destes animais em cativeiro requer conhecimento de seus hábitos e necessidades particulares, o que não ocorre na maioria dos casos. Devido à falta de conhecimento ou ausência de estrutura necessária, muitos animais acabam por desenvolver distúrbios relacionados à alimentação, estímulos ambientais, temperatura, substrato ou ambiente inadequados em que são mantidos. O presente trabalho teve por objetivo relatar o caso de uma penectomia e celiotomia exploratória em um jabuti (Chelonoidis carbonarius) cativo, que apresentava apatia, anorexia e sinais clínicos compatíveis com prolapso peniano.

Palavras-chave: Estase intestinal. Jabuti-piranga. Prolapso peniano. 


\section{INTRODUÇÃO}

Os jabutis pertencem a Classe Reptilia, Subclasse Anapsida, Ordem Testudines e Família Testudinidae. São répteis de hábitos terrestres, corpo robusto e membros locomotores fortes, que suportam seu casco pesado e são adaptados para caminhar em ambientes rústicos (CUBAS; BAPTISTOTTE, 2007). Estão distribuídos em países tropicais como Equador, Guianas, Paraguai, Venezuela, Caribe e Brasil, onde habitam as espécies jabuti-piranga (Chelonoidis carbonarius), jabuti-tinga (Chelonoidis denticulata) e jabuti-argentino (Chelonoidis chilensis) (PRITCHARD, 1979).

O jabuti-piranga é um réptil onívoro, alimentando-se de vários tipos de verduras, folhas, frutos, flores e proteína animal, como pequenos vertebrados, insetos, minhocas e carne. A manutenção desta espécie em cativeiro vem sendo uma prática cada vez mais observada no Brasil, tanto por mantenedores de fauna quanto por proprietários que os possuem como pet (DOS SANTOS; PEREIRA, 2011). Devido às necessidades especiais requeridas por esta espécie, como temperatura, alimentação, substrato e ambiente adequados, os erros de manejo estão frequentemente relacionados às afecções que levam os jabutis ao atendimento em clínicas e hospitais veterinários (CARVALHO, 2004). Distocia, prolapso peniano, prolapso de cloaca, cólon, bexiga e oviduto, hiper e hipovitaminoses e distúrbios alimentares são enfermidades frequentemente descritas (AQUILAR et al., 2006). A ingestão de corpos estranhos também é relatada nestas espécies, devido à prática de geofagia, o que pode acarretar em obstrução e lesão do trato gastrointestinal. (BJORNDAL, 1997; MATUSHIMA, 2001). Deste modo, o presente relato de caso tem como objetivo relatar o caso de um jabuti com prolapso peniano e suspeita clínica de impactação intestinal.

\section{RELATO DE CASO}

Um jabuti-piranga (Chelonoidis carbonarius), adulto, macho, pesando $4,84 \mathrm{~kg}$, foi encaminhado ao Hospital de Clínicas Veterinárias da Universidade Federal do Rio Grande do Sul apresentando prolapso peniano e anorexia. Ao exame clínico foi observado prostração, mucosas normocoradas, edemaciação do olho esquerdo, desidratação moderada e prolapso peniano com presença de necrose. Previamente ao encaminhamento, o animal estava recebendo curativos a base de açúcar cristal e enrofloxacina ( $5 \mathrm{mg} / \mathrm{kg}, \mathrm{SID}, 7$ dias). Foram 
realizados exames hematológicos, cujos resultados encontravam-se dentro dos padrões fisiológicos para a espécie, e radiográficos, sendo observado, neste último, trato gastrointestinal repleto de material radiopaco, com suspeita de fecaloma (Figura 1).

Foi instituída terapia com enrofloxacina $10 \mathrm{mg} / \mathrm{kg}$ (SID, IM, 8 dias), meloxicam 0,3 mg/kg (SID, IM, 7 dias) e fluidoterapia com solução de Ringer com lactato 20 ml/kg (BID, SC, 3 dias). O animal foi encaminhado para procedimentos cirúrgicos de penectomia e esofagostomia. Como medicação pré-anestésica foi utilizado morfina $2 \mathrm{mg} / \mathrm{kg}$, midazolam $1 \mathrm{mg} / \mathrm{kg}$ e cetamina $10 \mathrm{mg} / \mathrm{kg}$, todos por via intramuscular. Realizou-se o acesso venoso da veia jugular e indução anestésica com propofol $10 \mathrm{mg} / \mathrm{kg}$, intravenoso. $\mathrm{O}$ animal foi intubado com tubo endotraqueal 2,5 , sem cuff e a manutenção do plano anestésico obtida com isoflurano vaporizado com oxigênio $100 \%$ ao efeito. Foi administrado enrofloxacina como profilaxia antimicrobiana (10 mg/kg, IV) e a frequência e ritmo cardíaco foram monitorados por eletrocardiografia. Para o bloqueio epidural foi administrado bupivacaína $1 \mathrm{mg} / \mathrm{kg}$ (Figura 1A). A antissepsia do pênis e cloaca foi realizada com solução de clorexidine 4\% (Figura 1B), os corpos cavernosos foram ligados individualmente, em massa, com fio de sutura de polipropileno 3-0, a incisão foi realizada proximal à área necrótica, removendo todo tecido desvitalizado, seguido de sutura em bolsa de tabaco na cloaca (Figura 1C).
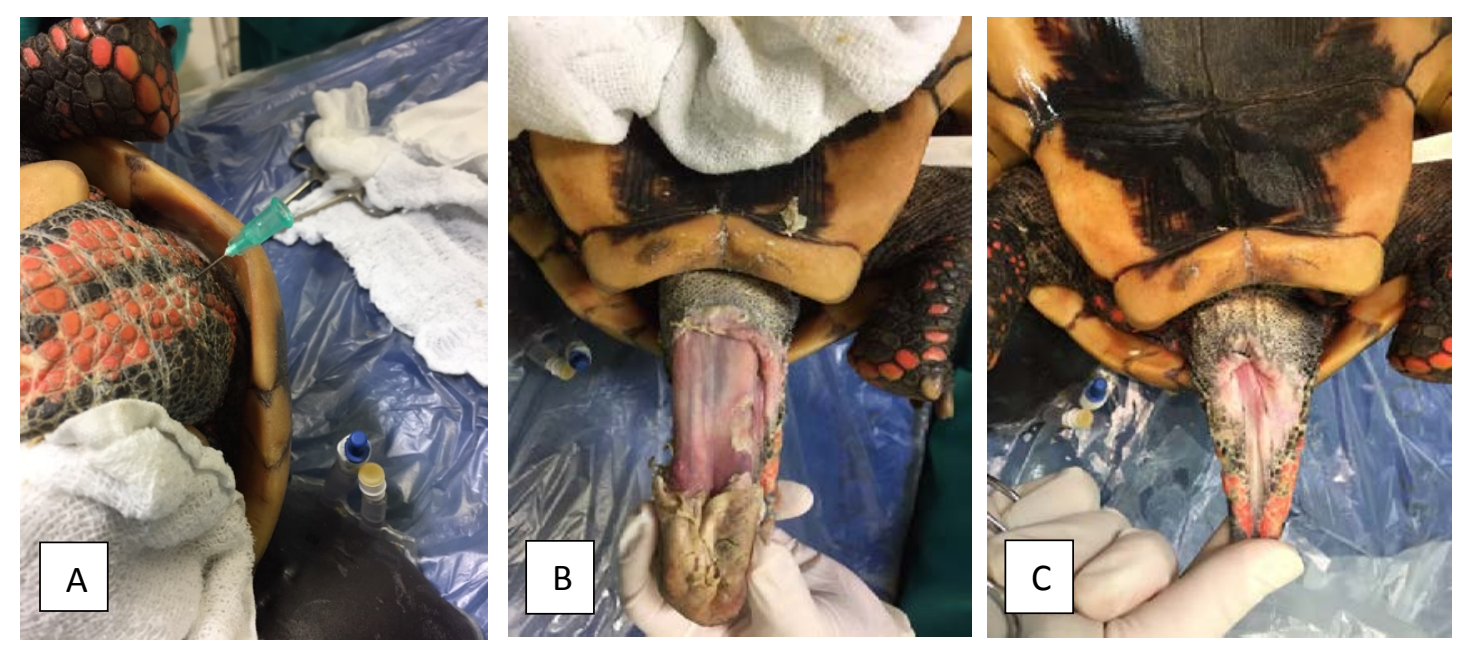

Figura 1 - Bloqueio epidural (A), assepsia de pênis e cloaca (B) e aspecto final do procedimento de penectomia realizado em Chelonoidis carbonarius (C). Fonte: Arquivo pessoal. 
Em seguida, foi realizado procedimento de esofagostomia cervical para fornecer uma via de alimentação e administração de medicamentos ao animal. No pós-operatório foi iniciada administração de meperidina $10 \mathrm{mg} / \mathrm{Kg}$ (SID, IM, 7 dias). Óleo mineral $5 \mathrm{ml}$ (BID, VO, 3 dias), simeticona (75 mg/ml) $1 \mathrm{ml}$ (BID, VO, 3 dias), solução de cloreto de sódio 0,9 \% $100 \mathrm{ml}$ (BID, 3 dias) e suplemento vitamínico e mineral 0,5 ml/kg (BID, 4 dias), todos via sonda esofágica. Foi realizado novo exame radiográfico para acompanhamento da impactação, sem evidências de alterações do quadro quando comparado ao exame anterior. A administração da medicação via sonda esofágica foi interrompida quatro dias após o procedimento cirúrgico, pois o animal regurgitou todo o conteúdo anteriormente administrado.

Uma semana após a cirurgia o paciente apresentou dispneia e bradicardia severas, sendo necessárias oxigenoterapia, fluidoterapia com solução de Ringer com lactato $(20 \mathrm{ml} / \mathrm{kg})$, além de monitoramento constante através de observação e aferição de parâmetros, como frequência respiratória, cardíaca e reflexos. Neste mesmo dia, foram drenados $120 \mathrm{ml}$ de conteúdo gástrico através de sonda esofágica. Como até esse momento o paciente não havia urinado nem defecado, além de não ter se alimentado, mesmo após conduta clínica medicamentosa de estímulo gastrointestinal, associado à observação das imagens dos exames radiográficos (Figura 2), sugestivas de impactação intestinal, optou-se por uma nova intervenção cirúrgica.
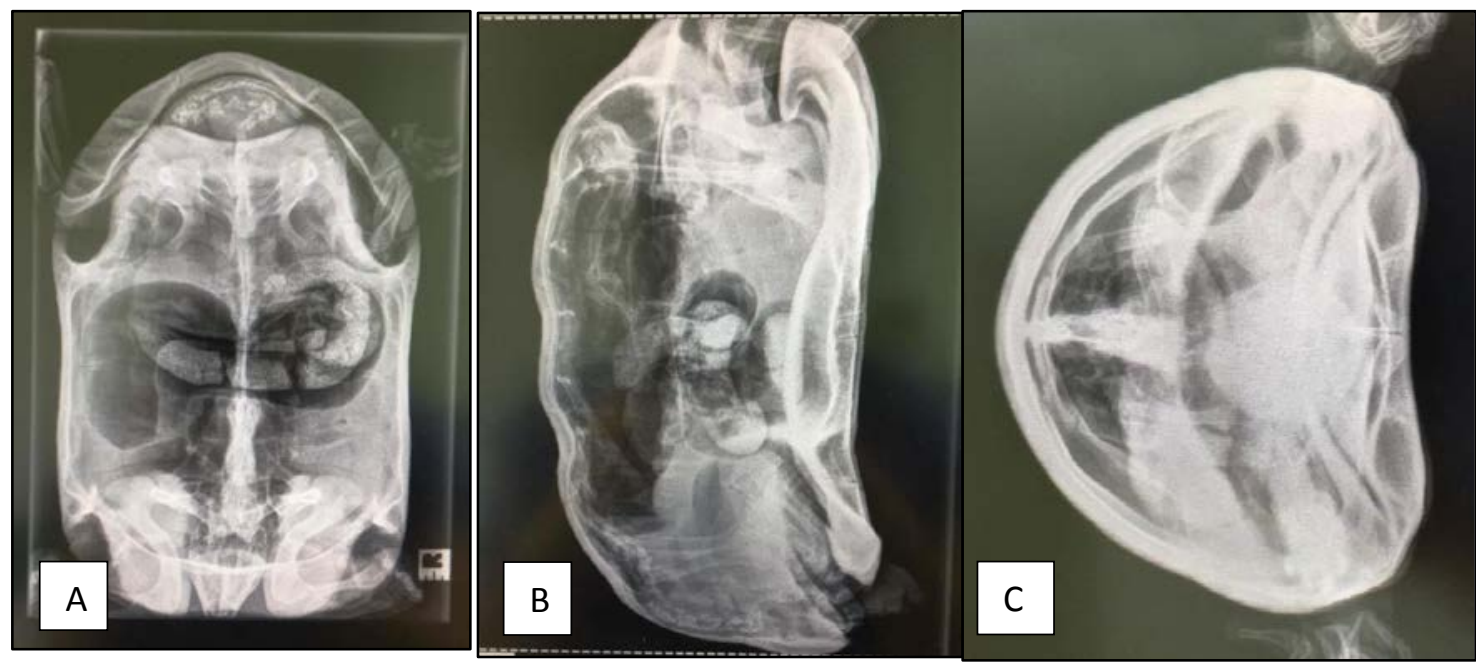

Figura 2 - Projeção radiográfica ventro-dorsal (A), projeção radiográfica latero-lateral direita (B) e projeção radiográfica crânio-caudal em Chelonoidis carbonarius (C). Fonte: Arquivo pessoal. 
Após estabilização do paciente, o mesmo foi encaminhado para procedimento cirúrgico de celiotomia exploratória. Como medicação pré-anestésica, foi utilizada morfina $(2 \mathrm{mg} / \mathrm{kg})$, midazolam (1 mg/kg) e cetamina (10 mg/kg), todos por via intramuscular e, para indução, propofol (10 mg/kg, IV). Após indução, foi realizada intubação orotraqueal com tubo endotraqueal 2,5, sem cuff, com respiração assistida e controle de frequência e ritmo cardíaco através de eletrocardiografia. A manutenção anestésica de todo procedimento foi realizada com isoflurano, vaporizado em oxigênio 100\%. Foi administrado ceftriaxona (50 $\mathrm{mg} / \mathrm{kg}$, IV) como profilaxia antimicrobiana. A antissepsia foi realizada com álcool $70 \%$ e solução de clorexidine 4\%. A osteotomia do plastrão foi realizada (Figura 3A) utilizando-se serra oscilatória e lâmina de $3 \mathrm{~cm}$. O plastrão foi irrigado com solução fisiológica durante o corte para dissipar o calor resultante do ato de serrar e para facilitar a remoção do pó de osso. Com um elevador periosteal, foi elevado o fragmento cortado e separada a musculatura peitoral e pélvica do periósteo (Figura 3B). Foi feita incisão na membrana celomática para acesso à cavidade interna e exploração do sistema gastrointestinal e urinário (Figura 3C).

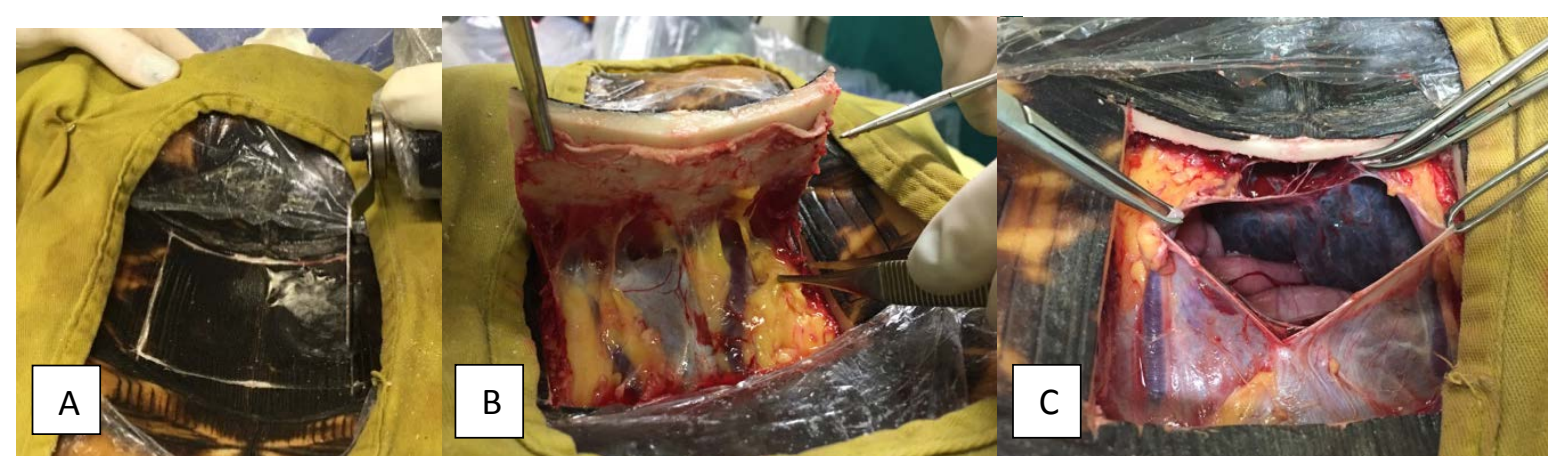

Figura 3 - Procedimento de celiotomia realizado em Chelonoidis carbonarius. Abertura do plastrão (A), retirada de fragmento do plastrão (B) e acesso à cavidade celomática (C). Fonte: Arquivo pessoal.

Foram observados fecalomas móveis e fragmentados dentro de todo intestino e bexiga urinária extremamente repleta e distendida. Muitas estruturas não foram visualizadas nem exteriorizadas da cavidade devido à presença de aderências entre alças intestinais e a abertura do plastrão ser limitada. Ao final do procedimento, foi realizada sutura da membrana celomática em padrão simples contínuo com fio monofilamentar não absorvível 
3-0. O plastrão e seu respectivo fragmento retirado foram perfurados, de modo a formar dois furos de cada lado da superfície serrada, totalizando 16 furos, quatro em cada superfície do quadrilátero, utilizando furadeira e broca $2 \mathrm{~mm}$. Para fixação do plastrão utilizou-se fio de aço monofilamento número 1-0 (Figura 4A). Após o fechamento, os bordos foram cobertos com adesivo hipoalergênico para evitar contato das porções ósseas seccionadas (Figura 4B) com o acrílico autopolimerizante que foi utilizado para fechamento final (Figura 4C).
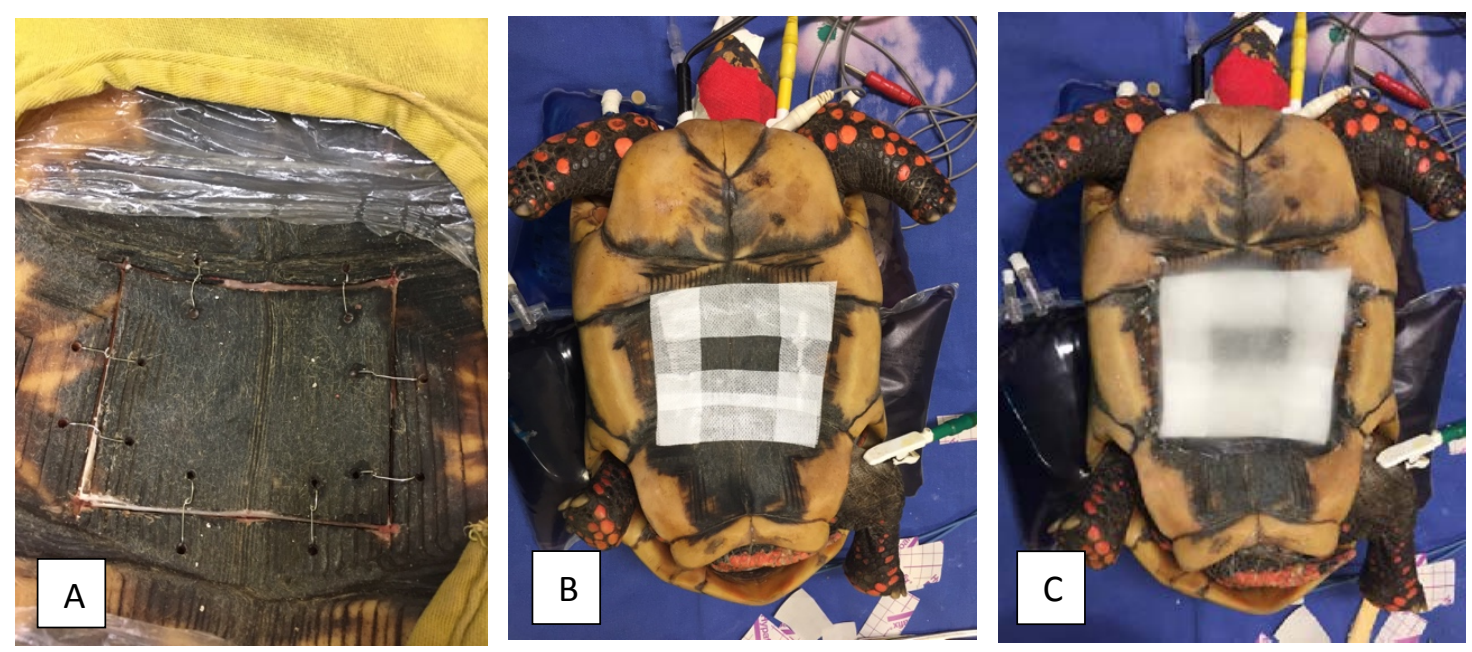

Figura 4 - Fixação do plastrão após celiotomia realizada em Chelonoidis carbonarius (A), cobertura dos bordos com adesivo hipoalergênico (B), fechamento final com acrílico autopolimerizante (C). Fonte: Arquivo pessoal.

Ao final do procedimento, o animal voltou a respirar de forma espontânea, porém mantendo a frequência respiratória e cardíaca baixas. Ficou sob monitoramento intensivo, sendo aferida frequência respiratória e cardíaca, além de reflexos, durante toda noite e manhã do dia seguinte, quando o animal foi a óbito.

\section{RESULTADOS E DISCUSSÃO}

Após análise do histórico do paciente, associado ao exame físico e exames complementares, chegou-se à conclusão de que o mesmo apresentava prolapso peniano com necrose já evidente. O prolapso peniano em jabutis ocorre por trauma resultante do atrito do pênis com uma superfície de contato, devido à exposição do pênis, que pode ocorrer por acidentes no momento da cópula, afecções neurológicas, hiperparatireoidismo secundário nutricional, separação forçada durante a cópula, inflamação, infecção, trauma, cálculo 
vesical ou cloacal e parasitas intestinais (RAMOS et al., 2009). Após lesão inicial, rapidamente o tecido lesado se torna edemaciado impedindo seu retorno à cloaca. Com esta exposição constante o órgão torna-se sujeito a novos traumas e infecções secundárias. A evolução do quadro pode levar à necrose de pênis e da cloaca, agravando o prognóstico do paciente (RAMOS et al., 2009). O tratamento conservativo é inicialmente recomendado, utilizando-se de técnicas para redução do edema, evitando, deste modo, a necessidade de submeter o paciente a um procedimento cirúrgico e anestésico (BENNETT; MADER, 2006). Neste caso, o tratamento conservativo de escolha foi realizado com açúcar cristal, objetivando a diminuição do edema e uma ação antimicrobiana (SERAFINI et al., 2012). A penectomia foi a técnica de última escolha, utilizada para amputação do pênis, realizada de acordo com a viabilidade dos tecidos envolvidos, após insucesso do tratamento conservativo (CUBAS; BAPTISTOTTE, 2007; DUTRA, 2014).

Foi relatado que o animal não se alimentava, defecava ou urinava desde o dia em que foi detectado o prolapso peniano. Sendo assim, optou-se por realizar esofagostomia no mesmo dia do procedimento cirúrgico de penectomia, seguindo técnica utilizada para pequenos animais e também aplicada para jabutis, levando-se em consideração as diferentes anatomias, sendo igualmente satisfatório seu desenvolvimento e resultado (CUBAS; BAPTISTOTTE, 2007; DUTRA, 2014). Com o objetivo de criar uma via de fácil acesso para fornecer ao paciente alimentação forçada, terapia medicamentosa e fluidos, visando o reestabelecimento de suas funções fisiológicas, a técnica de esofagostomia foi realizada com sucesso, confirmada através de exame radiográfico posterior.

Após o procedimento cirúrgico, foi observado, ao exame radiográfico, trato gastrointestinal repleto de material amorfo e radiopaco, o que, juntamente com o histórico do animal, levou à suspeita de impactação por fecaloma ou estase intestinal (SILVERMAN; JANSSEN, 1996). Para tal, foi realizada terapia medicamentosa com analgésico, estimulante para eliminação de gases, laxante e fluído, todos via sonda esofágica, com o intuito de lubrificar o conteúdo do intestino e permitir que o mesmo pudesse ser deslocado até a porção final do reto e ser expelido (BICHARD; SHERDING, 2003). Entretanto, o animal manteve-se sem apetite, sem defecar e urinar, além de ter regurgitado o conteúdo administrado. Optou-se, então, por 
novo procedimento cirúrgico, através de celiotomia exploratória (KIRCHGESSNER; MITCHELL, 2009). A celiotomia é indicada em afeç̧ões nos sistemas reprodutivo, urinário e gastrointestinal, como distocia, ruptura de ovos, cálculo cístico e obstrução ou torção intestinal (KIRCHGESSNER; MITCHELL, 2009). No caso relatado, o acesso foi realizado por osteotomia de plastrão, técnica traumática e precisa, que permite visualização dos órgãos presentes na cavidade. O local de eleição para a osteotomia foi nos escudos abdominais e femorais, evitando os ossos pélvicos, com incisão em formato de cunha, para que o fragmento retirado encaixe ao final do procedimento sem adentrar a cavidade celomática, conforme previamente relatado (BENNETT; MADER, 2006). O conteúdo gastrointestinal observado apresentava-se com boa consistência e mobilidade, sem excesso de gases ou distensão de paredes intestinais. Foi observada distensão vesical por excesso de urina retida, a qual foi drenada através de cistocentese e enviada para análise laboratorial por apresentar aspecto floculento. $\mathrm{O}$ exame de urina não é um teste fidedigno para tais animais, pois seu rim não concentra urina, tornando a avaliação de densidade limitada como marcador de função renal. Além disso, antes de entrar na bexiga, a urina passa através do urodeu da cloaca, contaminando-a (DUTRA, 2014). Deste modo, qualquer avaliação de sistema renal através de resultados da urinálise pode não ser representativa da realidade.

Ao final do procedimento foi realizado o fechamento da cavidade com resina acrílica a prova d'água e de secagem rápida para perfeita vedação do fragmento retirado (CUBAS; BAPTISTOTTE, 2007). A intervenção cirúrgica foi realizada segundo recomendações de Bennett e Mader (2006).

A correta composição de uma dieta balanceada para a espécie deve conter: $70-85 \%$ de vegetais, $10-20 \%$ de frutas e $5-10 \%$ de proteína animal (CUBAS; BAPTISTOTTE, 2007). O paciente em questão recebia $80-90 \%$ de frutas e verduras e $10-20 \%$ de proteína animal, predominantemente constituída de peixe, sendo uma proporção um pouco diferente do recomendado, sugerindo alteração do conteúdo do trato gastrointestinal.

Por serem ectotérmicos, a temperatura em que os répteis são mantidos influencia de maneira direta no seu estado geral de funcionamento do organismo (BENNETT; MADER, 2006). O paciente em questão era mantido em recinto ao ar livre sem fonte de calor ou 
controle de temperatura, o que também pode ter levado a alterações em seu comportamento, alimentação e fisiologia de maneira geral.

\section{CONCLUSÃO}

Ainda há muitos questionamentos relacionados à medicina de répteis. Esse grupo de animais possui peculiaridades diversas, extremamente diferentes de outros grupos, dificultando a tomada de decisão para uma conduta clínica e cirúrgica adequadas.

A conduta de escolha para o tratamento, neste caso, foi de acordo com dados os bibliográficos disponíveis. Entretanto, os fatores predisponentes do prolapso peniano, os elementos que originaram as aderências entre as alças intestinais e o quadro clínico do paciente, possivelmente atuaram de forma sinérgica, contribuindo para o óbito do animal.

\section{PENECTOMY AND EXPLORATORY CELIOTOMY IN Chelonoidis carbonarius (SPIX, 1824) - CASE REPORT}

\section{ABSTRACT}

abutis are reptiles with terrestrial habits that belong to the Order Chelonia, Family Testudinidae, and are distributed all over tropical countries, including Brazil. The redfooted tortoise (Chelonoidis carbonarius) stands out as a species found in captivity, either because of the need to maintain individuals who cannot be returned to the wild environment or because it is kept as a pet. Keeping these animals in captivity requires knowledge of their particular habits and needs, which does not occur in most cases. Due to lack of knowledge or lack of necessary structure, a lot of animals end up developing disorders related to inadequate feeding, environmental stimuli, temperature, substrate or environment where they are maintained. The present study had the objective to report the case of a penectomy and exploratory celiotomy in a captive tortoise (Chelonoidis carbonarius), who presented apathy, anorexia and clinical signs of penile prolapse.

Keywords: Intestinal stasis. Red-footed tortoise. Penile prolapse. 


\section{PENECTOMÍA Y CELIOTOMÍA EXPLORATORIA EN Chelonoidis carbonarius (SPIX, 1824) - INFORME DE CASO}

\section{RESUMEN}

as tortugas, son reptiles de hábitos terrestres, que están distribuidos por diversos países tropicales, habitando también Brasil. La Tortuga-piranga (Chelonoidis carbonarius) se destaca como especie encontrada en cautiverio, sea por necesidad de mantenimiento de individuos que no pueden volver a la vida libre o por propietarios particulares que los mantienen como animales de compañía. El mantenimiento de estos animales en cautiverio requiere conocimiento de sus hábitos y necesidades particulares, lo que no ocurre en la mayoría de los casos. Debido a la falta de conocimiento o ausencia de estructura necesaria, muchos animales acaban desarrollando disturbios relacionados con la alimentación, estímulos ambientales, temperatura, sustrato o ambiente inadecuados en que se mantienen. El presente trabajo tuvo como objetivo relatar un caso de una penectomia y celiotomia exploratoria en una Tortuga-piranga (Chelonoidis carbonarius) en cautiverio, que presentaba apatía, anorexia y signos clínicos compatibles con prolapso peniano.

Palabras clave: Estase intestinal. Tortuga-piranga. Prolapso peniano.

\section{REFERÊNCIAS}

AQUILAR, R.; DIVERS, S. M. H.; DIVERS, S. J. H. Atlas de Medicina Terapêutica e Patologia de Animais Exóticos. São Caetano do Sul: Interbook, 2006. P. 375.

BICHARD, S. J; SHERDING, R. G. Manual Saunders - Clínica de Pequenos Animais. 2. ed. São Paulo: Roca, 2003.

BJORNDAL, K. A. Foraging ecology and nutrition of sea turtles. In: LUTZ, P. L.; MUSICK, J. A. The Biology of Sea Turtles. Florida: CRC Press, 1997. P. 199-231.

CARVALHO, R. C. Topografia vértebro-medular e anestesia espinhal em jabuti das patas vermelhas Geochelone carbonaria (SPIX, 1824). São Paulo: USP, 2004. 126p. Dissertação (Mestrado em Ciências Veterinárias), Programa de Pós-Graduação em Anatomia dos Animais Domésticos e Silvestres, Faculdade de Medicina Veterinária e Zootecnia, Universidade de São Paulo, 2004.

CUBAS, P. H.; BAPTISTOTTE, C. Chelonia (tartaruga, cágado, jabuti). In: CUBAS, Z. S.; SILVA, J. C. R.; CATÃO-DIAS, J. L. Tratado de Animais Selvagens. São Paulo: Roca, 2007. P. 86-103. 
DUTRA, G. H. P. Testudines (Tigra d'água, Cágado e Jabuti). In: CUBAS, Z. S.; SILVA, J. C. R.; CATÃO-DIAS, J. L. Tratado de Animais Selvagens. 2. ed. São Paulo: Roca, 2014. P. 256-294.

KIRCHGESSNER, M.; MITCHELL, M. A. Chelonians. In: MITCHELL, M. A.; TULLY JR, T. N. Manual of Exoctic Pet Practice. Missouri: Saunders Elsevier, 2009. P. 243-244.

BENNETT, R. A.; MADER, D. R. Cloacal Prolapse. In: MADER, D. R. Reptile Medicine and Surgery. 2 ed. Missouri: Saunders Elsevier, 2006. P. 751-755.

MATUSHIMA, E. R. Chelonian infectious diseases and general medicine. In: FOWLER, M. E.; CUBAS, Z. S. Biology, Medicine and Surgery of South America Wild Animals. Ames: lowa State University Press, 2001. P. 22-24.

PRITCHARD, P. C. H. Encyclopedia of turtles. New Jersy: TFD, 1979. P.326-323.

RAMOS, R. M.; VALE, D. F. V.; HANAWO, M. E. O. C.; et al. Penectomia em caso de prolapso peniano em Jabuti-piranga (Geochelone carbonaria) - Relato de caso. Jornal Brasileiro de Ciência Animal, v. 2, n. 3, p. 166-174, 2009.

DOS SANTOS, G. J.; PEREIRA, R. E. P. Levantamento de Aspergillus fumigatus e Strongyloides sp. em jabutis mantidos em cativeiro no bosque municipal Dr. Belírio Guimarães BrandãoZoológico Municipal da cidade de Garça-SP. Revista Científica Eletrônica de Medicina Veterinária, ano IX, n. 16, p. 01-29, 2011.

SERAFINI, G. M. C.; SCHOSSLER, J. E. W.; AMARAL, A. S.; et al. Açúcar granulado ou em gel no tratamento de feridas em cães. Ciência Rural, v. 42, n. 12, p. 2213-2218, 2012.

SILVERMAN, S.; JANSSEN, D. L. Diagnostic Imaging. In: MADER, D. R. Reptile Medicine and Surgery. Philadelphia: Saunders, 1996. P. 258-264. 